\title{
Odd-even effect of melting finite polymer film on square lattice
}

\author{
Tieyan Si \\ Academy of Fundamental and Interdisciplinary Sciences, \\ Harbin Institute of Technology, Harbin, 150080, China
}

(Dated: April 2, 2018)

\begin{abstract}
Two dimensional film system bears many exotic thermodynamics behaviors. We proposed a mathematical physics model to explore how the melting temperature of a two dimensional mathematical dimer film depends on the odd-eveness of the finite width of dimer film. A weak external bond between dimers is introduced into the classical dimer model in this dimer film. We derived a general equation of melting temperature and applied it for computing the melting temperature of a dimer film covering a finite square lattice. The melting temperature is proportional to the external bonding energy that we assume it binds neighboring dimers together and proportional to the inverse of entropy per site. Further more, it shows fusing two small rectangular dimer film with odd number of length into one big rectangular film gains more entropy than fusing two small rectangles with even number of length into the same big rectangle. Fusing two small toruses with even number of length into one big torus reduces entropy. Fusing two small toruses with odd number of length increases the entropy. Thus two dimer films with even number of length repel each other, two dimer films with odd length attract each other. The odd-even effect is also reflected on the correlation function of two topologically distinguishable loops in a torus surface. The entropy of finite system dominates odd-even effect. This model has straightforward extension to longer polymers and three dimensional systems.
\end{abstract}

PACS numbers: 68.60.Dv; 68.55.am; 65.40.gd; 64.70.qd; 64.70.dj.

\section{INTRODUCTION}

The melting of two dimensional solid film, such as polymer film, liquid-crystal film, vortex film, was theoretically suggested by beginning with dissociation of vortex pairs or topological defect [1]. In recent years, a multiplestep melting behavior of two dimensional liquid-crystal film was experimentally observed [2]. Unlike the three dimensional system, the odd-even effect in two dimensional system is more subtle and hard to implement by practical equipment. When chemist study the miscibility of binary mixtures of several low molar mass nematogens with a main chain liquid crystal polymer (TPB-x), it was found only when the length of the flexible alkyl spacers in liquid crystal polymer is odd, the binary mixtures are miscible. If the length of the flexible alkyl spacers is even, the mixtures of several low molar mass nematogens is immiscible 3]. The odd-even effect is also illustrated by the melting temperature of n-alkanes $\mathrm{C}_{n} \mathrm{H}_{2 n+2}$ [4]. The melting point of fatty acids with odd number of chain length are below its neighboring even number of chain length [4]. When the length of the n-alkanes increases by one unit from an even number to its next neighboring odd number, the melting temperature increases by a relatively smaller value compared with the case that from an odd number to even number [5] [6]. This odd-even effect is diminished when the chain length becomes longer than 16. The experiments believed it is the different crystalline structures of the odd and even oligomers that results in the odd-even effect and anomalous odd-even effect [3] [4] [5] [6]. A powerful Monte Carlo computer simulation of n-alkanes reported the odd-even dependence of the triple point temperature on the length of n-alkanes molecules [7] as well as some ordered phases of a lattice-gas systems of dimers, trimers, and tetramers [8] 9]. There is still no exact numerical simulation that could matche exactly the experimental data of melting temperature of three dimensional powder of polymers with different length.

Inspired by the experimentally observed odd-even phenomena above, we proposed an exact theoretical model for computing the melting temperature of polymer film since there is still no such an exact equations for computing the meting temperature so far. For a straightforward physical understanding on the odd-even effect of melting polymer by exactly solvable mathematical physics model, we study how the melting temperature of a dimer film covering a square lattice depends on the finite width of the dimer film.

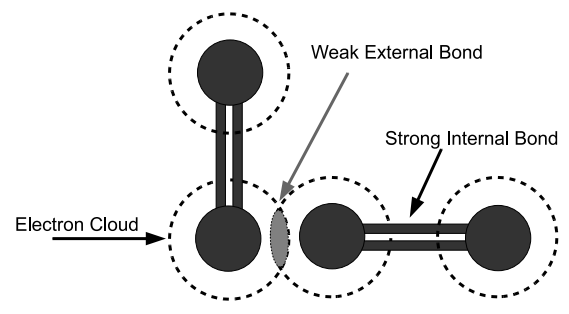

FIG. 1: We assumed there exists a weak external bond(the overlapping zone) between dimer molecules due to the fluctuating overlap of electron cloud. The internal bond is chemical bond which is a thousand times stronger than the external bond. This weak external bond could be estimated by the familiar van der Waals interacton between molecules which decays following $1 / r^{6}$.

As there is no report of experimental evidence on the order of phase transition about melting two dimensional 
film, we take a naive assumption that melting two dimensional dimer film is a first order phase transition. Computing the temperature requires an exact calculation of internal energy difference and entropy difference. The internal energy difference is straightforward to calculate. The entropy difference can be computed by classical dimer model. Classical dimer model counts how many different ways to cover a graph by pairing up two neighboring vertex [10]. Kasteleyn found a method to compute the exact number of dimer covering on square lattice [11]. The dimer configurations has a theoretical correspondence with fluctuating string configuration on two dimensional lattice [12]. There is no external bonding between dimers in classical dimer model except that the overlap of two dimers is forbidden, while here we introduced a weak external bond that binds neighboring dimers together in this dimer film model to simulate the melting phase transition(Fig. 1). The odd-even effect showed by this exact mathematical physics model is different from that of three dimensional polymer powders. The entropy variation for fusing two finite films into one or dividing one film into two also shows odd-even effect. As all know, cell division is a crucial process for life. While the liquid crystal membrane model had successfully predicted many properties of the biconcave shape of red blood cell [13]. The stable existence of the torus of liquid crystal membrane was first predicted by Helfrich's theory of lipid blayer membranes 14] and was confirmed by experiment later [15]. The second law of thermal dynamics holds both for liquid crystal film and polymer film. Thus we focus on the fundamental physical principal of film fusion or division without a specific molecules. The entropy principal for the odd-even effect of finite film division is also a fundamental principal for understanding many dynamic phenomena in other physical system.

The article is organized as following: In the section II, we proposed the thermodynamic equation for computing the melting temperature of dimer film on a constant area. The width of the rectangle varies from $\mathrm{N}$ to $\mathrm{N}+1$. In the section III, we take an infinitely long dimer film with finite width, and study how the entropy growth rate behaves when the width increases at small step. In section IV, we study the entropy difference when two small rectangles fuse into one big rectangle and when two small torus fuse into a big torus. In section $\mathrm{V}$, the correlation function of two loops on torus is computed to check its odd-even phenomena. The last section is a summary.

\section{THE MELTING TEMPERATURE OF DIMER FILM ON FINITE RECTANGULAR LATTICE WITH CONSTANT AREA}

The melting temperature of polymer is the temperature at which a crystalline of polymers transforms into a solid amorphous phase. As a mathematical modeling of polymer melting transformation, we first define the frozen phase and the melted phase of dimer film. The frozen phase is highly ordered phase, all dimers are oriented in the Y-direction. None of the dimers can rotate to $\mathrm{X}$-direction. The total number of dimer configuration in the frozen phase is just one. The melted phase is a disorder phase, the dimer at each lattice site can transform from a X-oriented dimer into a Y-oriented dimer or vice versa. All possible different configurations of dimer covering should be taken into account. The internal energy difference between the frozen phase and the melted phase is counted by the number of weak external bond that we assume it binds neighboring dimers. In the frozen phase, these weak external bond fixed the orientation of dimers. In the melted phase, these weak external bond is broken by thermal energy. As this dimer film is the simplest modeling of the melting process, we assume it is a first order phase transition since there is still no experimental results of melting two dimensional dimer film so far. The first order phase transition requires that the wellknown Gibbs free energy is zero at the transition point, $\Delta G=\Delta U-T \Delta S=0$.

The energy quanta for breaking a weak external bond is $\epsilon_{i}$. The index $i$ labels the position of the weak external bond on the dual lattice of dimer lattice which is denoted by the crossing stars in Fig. 2. The total thermal energy for breaking the dual lattice is $\Delta U=\sum_{i} \epsilon_{i}$. Denoting the partition function of the frozen phase as $Z_{1}$ and the partition function of the melted phase as $Z_{2}$, the melting temperature reads

$$
T_{c}=\frac{\Delta U}{\Delta S}=\frac{\sum_{i} \epsilon_{i}}{k_{B} \log \left[Z_{2}\right]-k_{B} \log \left[Z_{1}\right]} .
$$

$k_{B}=1.3806488 \times 10^{-23} \mathrm{~m}^{2} \mathrm{Kgs}^{-2} \mathrm{~K}^{-1}$, is the Boltzmann constant. This equation holds for different lattices in different dimensions. Here we only consider a two dimensional finite rectangle of square lattice with $\mathrm{m}$ rows and $n$ columns. The number of lattice sites is $m \times n$. The total number of internal bond and external bond is $(2 m n-n-m)$. The number of internal bonds within dimer is $m n / 2$. Thus the total number of external bonds is $\frac{3}{2} m n-n-m$. The thermal energy breaks the external bonds to melt the dime film. If every external bond possesses the same energy quanta $\epsilon_{0}$, the thermal energy absorbed by the dimer film at the temperature is $\Delta U=\left(\frac{3}{2} m n-n-m\right) \epsilon_{0}$.

In the frozen phase, the entropy is zero since the number of dimer configuration is only one. In the melting phase, all different dimer coverings on the rectangle lattice is possible, thus we apply Kasteleyn's method [11] to derive an exact counting. The determinant of Kasteleyn's adjacent matrix $K$ on square lattice reads [1]

$$
K=\prod_{k=1}^{n} \prod_{j=1}^{m}\left(2 \cos \left[\frac{\pi j}{m+1}\right]+2 i \cos \left[\frac{\pi k}{n+1}\right]\right) .
$$

Here $\mathrm{m}$ and $\mathrm{n}$ are both even number. The number of all possible configurations of dimer covering is $Z=\sqrt{\operatorname{det} K}$ 11]. For other cases, $m$ is odd or even and $n$ is odd 
or even, the determinant of Kasteleyn's adjacent matrix $K$ has similar formulation as Eq. (21) 11]. Kasteleyn's method was mapped into an equivalent free fermion model in quantum field theory [16] which provides a physical picture of dimer covering of fermion pairs. Two dimers have a hard-core repulsive interaction between them since they can not overlap each other.
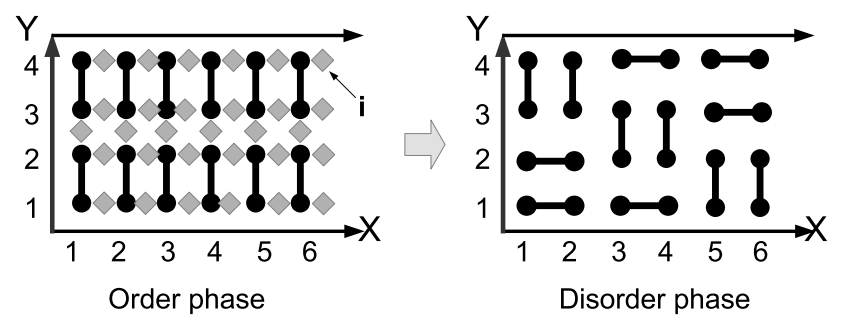

FIG. 2: The dimer film is approximate infinitely long. The width varies from 2 to 16 . The index $i$ indicates the position of weak external bonds. The grey cross star labels weak external bonding on the dual lattice of dimer lattice.

The odd-even effect is finite size effect, so we choose a finite square lattice with a large number of lattice sites of $m n=\Pi_{j=1}^{7} j \times 11 \times 13=720720$. The energy quanta of weak external bonding is taken as $\epsilon_{0}=10^{-4}$ for the convenience of computation. In the computation, the total number of lattice sites is invariant but the ratio of height to width varies. The width of the long rectangular belt $m$ runs from 2 to 16 . The length of this long rectangle at $\mathrm{n}=2$ is $(720720 / 2)$, it is almost infinitely long. The finite size effect is obvious for the width but is diminished in the lengthy direction. The output of Eq. (1) gives the melting temperature. The melting temperature increases as the width grows from $\mathrm{N}$ to $\mathrm{N}+\mathrm{l}$ (Fig. 3). When $\mathrm{N}$ grows larger than 16, the melting temperature approaches to a constant value. The odd-even effect is suppressed in thermal dynamic limit. The temperature curve connecting the odd number of width floats above that threads the even number of width. There is a similar but different phenomena in an experimental measurement of the melting temperature of n-alkanes $C_{n} H_{2 n+2}$ powder. The temperature curve connecting the odd number of length of $C_{n} H_{2 n+2}$ is below that for the even number of length of $C_{n} H_{2 n+2}[\underline{5}]$. Even though here we study the width dependence of dimer film which is different from the three dimensional experimental system, this theory maybe is still helpful for an exact understanding on the odd-even effect of polymer powders.

The temperature increase from a width of $\mathrm{N}$ to $\mathrm{N}+1$ also depends on the odd-evenness of the width number. The temperature increase from a width of $\mathrm{N}(\mathrm{N}$ is even number)to $\mathrm{N}+1$ is always bigger than that from a width of $\mathrm{N}+1$ to $\mathrm{N}+2$. For $\mathrm{N}=2$, the temperature increases by 0.175617 percent from a width of 2 to 3 . While it only increased 0.0561494 percent from a width of 3 to 4 . The temperature increase curve of $\Delta T_{c}$ that threads the even number of width is above that passing through the odd number of width (Fig. 4).

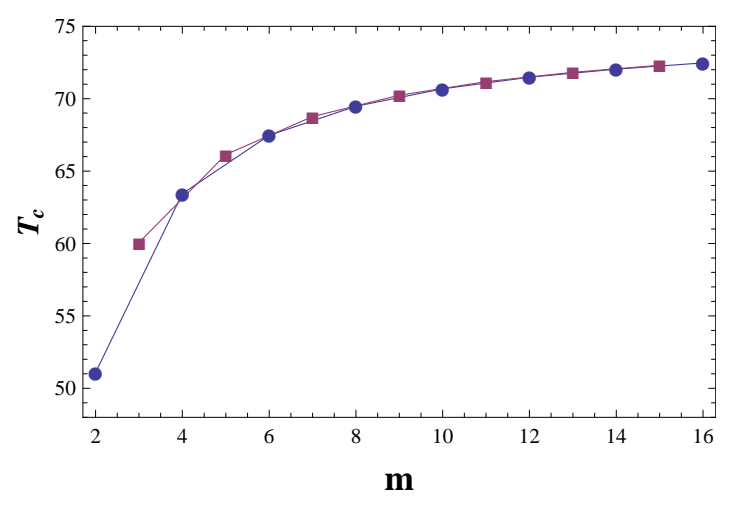

FIG. 3: The melting temperature of dimer film at different width from 2 to 16 . We take the unit energy of an external bond as $\epsilon_{0}=10^{-4}$ for simplicity.

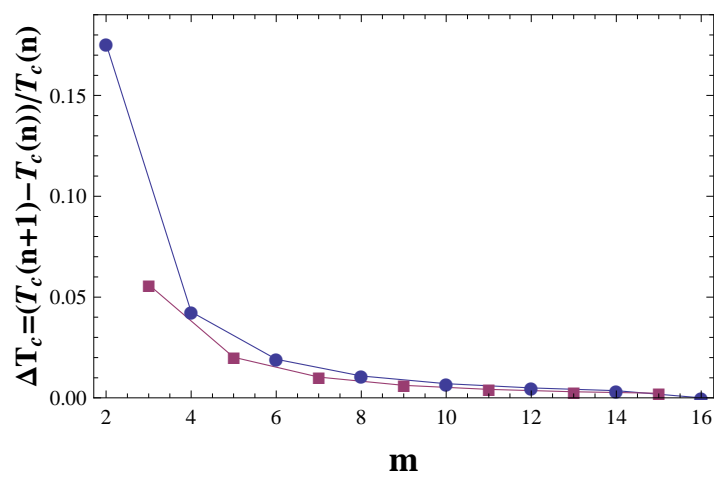

FIG. 4: The increase of melting temperature at each step where the width of the dimer film grows bigger by one unit.

The odd-even effect of melting temperature originates from the finite size effect of entropy. The entropy curve connecting the even number of width is above that connecting the odd number of width(Fig. 5). The entropy increase from $\mathrm{m}(\mathrm{m}$ is even) to $\mathrm{m}+1$ ( $\mathrm{m}$ is even) is negative. The entropy increase from from $\mathrm{m}$ to $\mathrm{m}+1$ becomes positive for an odd number of $\mathrm{m}$ (Fig. 5). If the long rectangle behaves like a water droplet which can vary its own shape following the second law of thermodynamics. There would be an entropy force when the rectangle reshapes itself. We define the entropy force induced as $F_{A}=T(\Delta S(m, n) / \Delta A)$, where the area of the film is $A=m n, T$ is temperature. The area is different for different width, so is the entropy covering the area. By this entropy force, we could predict the interaction of the film with its environment. Finite size effect determines the interaction between dimer film and its surrounding. 


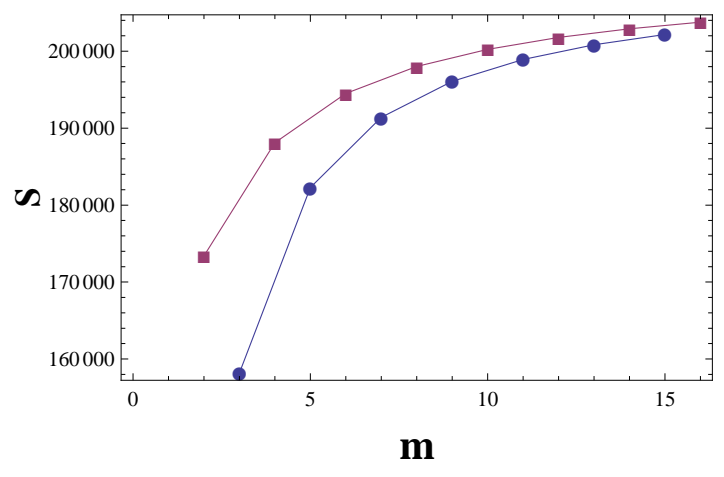

FIG. 5: The entropy of dimer coverings on the infinitely long rectangle. The total lattice sites of the rectangle is $\mathrm{mn}=\Pi_{i=1}^{7} i$ $\times 11 \times 13=720720$. The width of rectangle is represented by $m$.

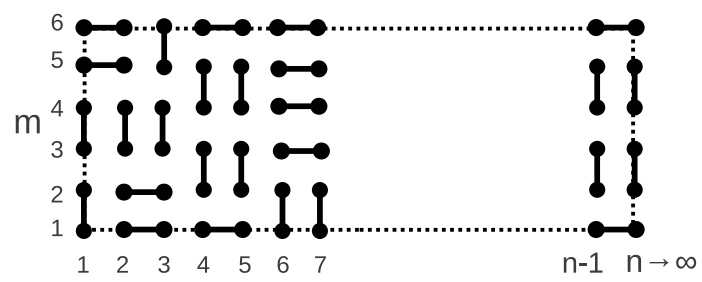

FIG. 6: The width of the dimer film is finite and is increasing step by step. The length of the dimer film would be infinitely long.

\section{THE MELTING TEMPERATURE OF DIMER FILM}

The melting temperature of dimer film with finite width approaches to a constant value in thermal dynamic limit. To explicitly express that constant value we study the entropy growth rate of an infinitely long rectangle when its length increases step by step (Fig. 6). The number of rows is fixed to a small value. The entropy of finite system is defined as the same formulation as thermodynamic entropy, $S=k_{B} \log [Z]$. Here Boltzmann constant $k_{B}$ is set to 1 for convenience. For fixed number of rows, the entropy growth for a growing number of columns by one unit reads

$$
\Delta S_{m, n}=k_{B} \log [Z(m, n)]-k_{B} \log [Z(m, n-1)] .
$$

The melting temperature is determined by the ratio of internal energy difference to entropy difference, $T_{c}=$ $\Delta U / \Delta S$. So entropy difference can be deduced by the melting temperature equation, $\Delta S=\Delta U / T_{c}$. The internal energy difference between $\mathrm{n}$ column and (n-1) column is $\Delta U=\left(\frac{3}{2} m n-n-m\right) \epsilon_{0}=N_{m, n} \epsilon_{0}$. The combination of $\Delta U$ and $\Delta S$ gives the entropy growth from $n$ column to $(n-1)$ column for a fixed number of $m$ rows,

$$
\Delta S_{m, n}=N_{m, n}\left(\frac{\epsilon_{0}}{T_{m, n}}-\frac{\epsilon_{0}}{T_{m, n-1}}\right)+\left(\frac{3}{2} m-1\right) \frac{\epsilon_{0}}{T_{m, n-1}} .
$$

According to the computation of melting temperature of long belt in last section, the temperature difference of $\Delta T_{c}=T_{c}(n)-T_{c}(n-1)$ for large number of $n$ approaches to zero. The first term at the right hand side of Eq. (4) approaches to zero when the number of columns goes to infinity, $\lim _{n \rightarrow \infty} N_{m, n}\left(\epsilon_{0} / T_{m, n}-\epsilon_{0} / T_{m, n-1}\right)=0$. As a result, the entropy increase now only depends on the number of rows,

$$
\Delta S_{m, n \rightarrow \infty}=\left(\frac{3}{2} m-1\right) \frac{\epsilon_{0}}{T_{m, n \rightarrow \infty}} .
$$

The right hand side of Eq. (5) is actually the derivative of entropy with respect to the number of columns in thermal dynamic limit. If we take a further derivative of $\Delta S_{m, n \rightarrow \infty}$ with respect to the number of rows $m$, it leads to constant $\kappa$,

$$
\kappa=\frac{\partial}{\partial m}\left[\lim _{n \rightarrow \infty} \frac{\Delta S}{\Delta n}\right]=\frac{3}{2} \frac{\epsilon_{0}}{T_{m, n \rightarrow \infty}} .
$$

As $(\Delta m \Delta n)$ indicates the area of an unit square on square lattice. $k$ is in fact the entropy per square. One square occupies four quarters of one lattice site. $k$ is also the entropy per site. The constant melting temperature in thermal dynamic limit now has a brief formulation,

$$
T_{n \rightarrow \infty}=\frac{3}{2} \frac{\epsilon_{0}}{\kappa} .
$$

This equation reveals the relationship between the melting temperature, entropy per site and external bonding energy. A stronger external bonding hold dimers together with more strength, thus it takes more thermal energy to melt. Melting process increases the entropy of dimer film to make it more chaos, so it also costs higher thermal energy to melt a highly ordered film with less entropy.

The external bonding energy can only be determined by experimental measurement. The entropy per site in the liquid phase of dimer film can be obtained by Kasteleyn's method. Here we derive the entropy per site by a different approach of Eq. (5). The linear dependence of $\Delta S_{m, n \rightarrow \infty}$ on the number of rows $m$ is verified by direct computation. We first computed the entropy growth rate for finite length and then extended the computation to infinite length. The computation is performed both for a rectangle and a torus. A rectangle has open boundary condition. A torus has periodic boundary condition in both the row's direction and the column's direction. Fig. 7 shows the entropy growth on a rectangle and a torus when the number of column increases from 2 to 20 . The number of rows is fixed to $m=10$. The entropy growth for the length growing from $n-1$ to $n$ (n is even) is always bigger than the case that $n$ is odd (Fig. 7). The entropy growth curve for an odd number of length $n$ increases as the length becomes longer. While the entropy growth curve connecting those even number of length decays. These two curves converges to the same constant value when the length of rectangle becomes bigger than 20. This phenomena also holds for 
torus, the convergence point of the two curves for torus is bigger than that of rectangle. It takes more steps for $\Delta S$ to converge on a torus than that on a rectangle. The limit value $\Delta S(n \rightarrow \infty, m=10)$ on rectangle is $\Delta S_{\text {rec }}(n \rightarrow \infty, m=10)=2.778441$, here we have made a cut off at $10^{-6}$. The limit value on a torus for large $n$ is a little bit higher, $\Delta S_{t o r}(n \rightarrow \infty, m=10)=2.969359$. The difference between the limit values of rectangle and torus vanishes for $m \rightarrow \infty$. Fig. 8 showed the limit values of $\Delta S_{t o r}(n \rightarrow \infty)$ and $\Delta S_{\text {rec }}(n \rightarrow \infty)$ when $m$ grows from 2 to 54 . Both the two limit values form a straight line.

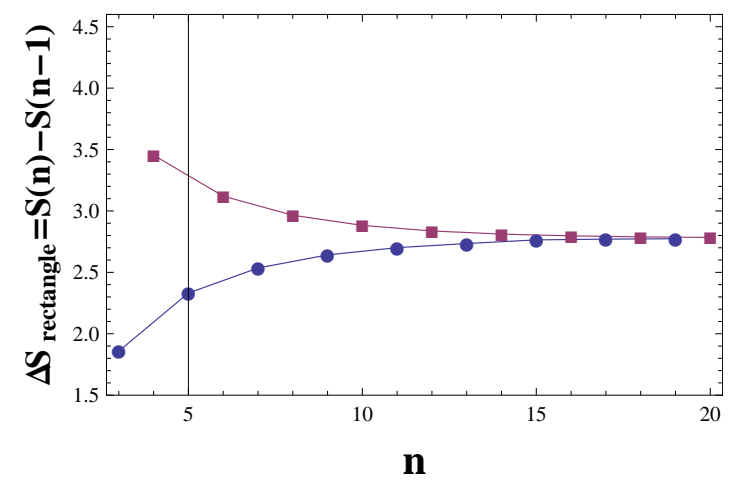

(a)

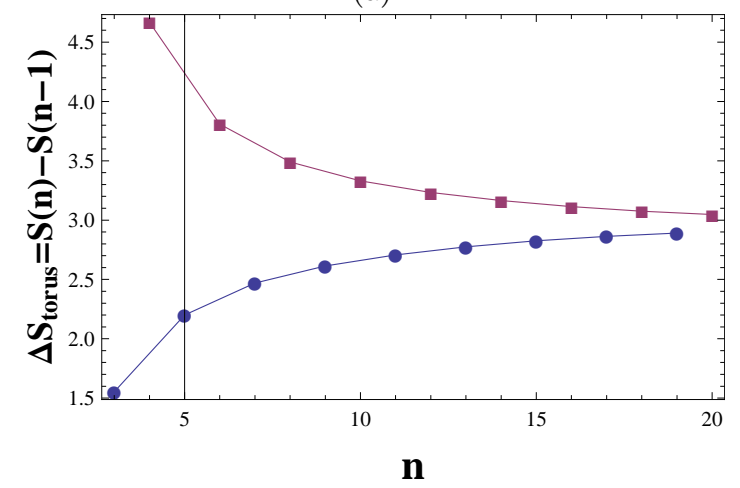

(b)

FIG. 7: (a) The entropy growth of a rectangle from odd number of columns to even number of columns, or vice verse. The Number of rows is fixed, $m=10$. (b)The entropy growth of a torus. The number of rows is kept to $m=10$. The number of columns varies from odd to even, or vice verse.

The limit values fit well with an empirical linear equation for large $m(m>100)$,

$$
\begin{aligned}
\Delta S_{r e c}(n \rightarrow \infty, m) & =0.29 m+C_{r e c}(m), \\
\Delta S_{\text {tor }}(n \rightarrow \infty, m) & =0.29 m+C_{\text {tor }}(m), \\
m & =2 k, \quad k=0,1,2,3, \cdots .
\end{aligned}
$$

The accuracy of the numerical values in equations are kept to the order of $10^{-2}$ for brevity without losing the key features. This empirical linear equation implies a constant slope,

$$
\kappa / k_{B}=\frac{\partial}{\partial m}\left[\lim _{n \rightarrow \infty} \frac{\Delta S}{\Delta n}\right]=0.29 .
$$

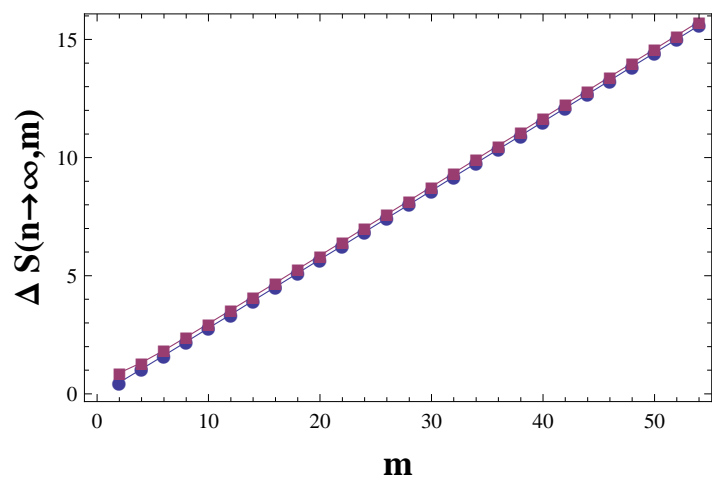

FIG. 8: The limit value of entropy growth for $n \rightarrow \infty$ at different number of rows $m$. The square dot represents torus. The disc dot represents rectangle. The limit value of torus is always higher than that of rectangle.

This is consistent with the computed temperature in Fig. (3) where $T_{c}$ approaches to a constant for large $m$. The probability of a dimer on square lattice obtained by Kasteleyn is 1.791622 [11]. The entropy per site reads $0.29=\log (1.791622) / 2$. This number is exactly the same as the slope of $\Delta S_{t o r}(n \rightarrow \infty)$ for large m. According to the melting temperature equation (77), the physical entropy per site for a dimer film on square lattice is $\kappa=0.29 \times k_{B}=0.400388152 \times 10^{-23} \mathrm{~m}^{2} \mathrm{Kgs}^{-2} \mathrm{~K}^{-1}$. The physical process of melting a real liquid crystal film experiences a multi-step transitions from an ordered phase into a liquid phase [2]. For instance, the liquid crystal film transform a crystal-B phase into a hexatic-B phase at $60{ }^{\circ} \mathrm{C}[2]$. As the temperature raises to $63{ }^{\circ} \mathrm{C}$, the liquid crystal film transform stepped into Sm-A phase 2]. It melts into an ordinary two dimensional liquid at about $66.3{ }^{\circ} \mathrm{C}[2]$. The melting temperature and external bonding energy quanta in Eq. (77) is not a quantity that can be determined by theoretical computations only, it can only be determined by experimental measurement. As this model does not focus on specific molecules, Eq. (17) also holds for liquid crystal film. Thus we simply assume the critical melting temperature is roughly $67^{\circ} \mathrm{C}$, i.e., $T_{c}=340 \mathrm{~K}$. Then we could estimate the external bonding energy,

$$
\epsilon_{0}=\frac{2}{3} \kappa T_{c}=0.907546778 \times 10^{-21} \mathrm{~m}^{2} \mathrm{Kgs}^{-2} .
$$

Thus the weak external bonding energy is about $0.907546778 \times 10^{-21} \mathrm{~J}$. As all know, $1 \mathrm{eV}=$ $1.602176565(35) \times 10^{-19} \mathrm{~J}$, thus the weak external bonding energy is about $\epsilon_{0}=0.566446169 \times 10^{-2} \mathrm{ev}$. While the usual Carbon-Carbon bond energy is around 3.60 3.69 ev. Since $\epsilon_{0}$ is much smaller than the chemical bond, we call it weak external bond.

The difference between $\Delta S_{\text {tor }}(n \rightarrow \infty)$ and $\Delta S_{\text {rec }}(n \rightarrow$ $\infty)$ is a function of $m, \Delta C(m)=C_{\text {tor }}(m)-C_{\text {rec }}(m)$. The maximal value of $\Delta C(m)$ is $\Delta C(2)=0.400162 . \Delta C(m)$ decays when $m$ grows. The most rapid decay occurs from $m=2$ to $m=50$. When $m$ continue to grow from 
$m=50$ to $m=500, \Delta C(m)$ only drops from 0.157040 to 0.149912 .

The topological difference between rectangle and torus can be characterized by their different speed of entropy growth,

$$
\Delta C(m)=\Delta S_{\text {tor }}(n \rightarrow \infty)-\Delta S_{\text {rec }}(n \rightarrow \infty) .
$$

The rapid decay of $\Delta C(m)$ is illustrated in Fig. 9, A logarithmic plot of $\Delta C(m)$ with respect to $\mathrm{m}$ still behaves as rapid decay instead of a straight line. The decaying rate of $\Delta C(m)$ is much faster than exponential decay. The topological difference between torus and rectangle vanishes when the width goes to infinity. The melting temperature in thermal dynamic limit does not depend on boundary condition.

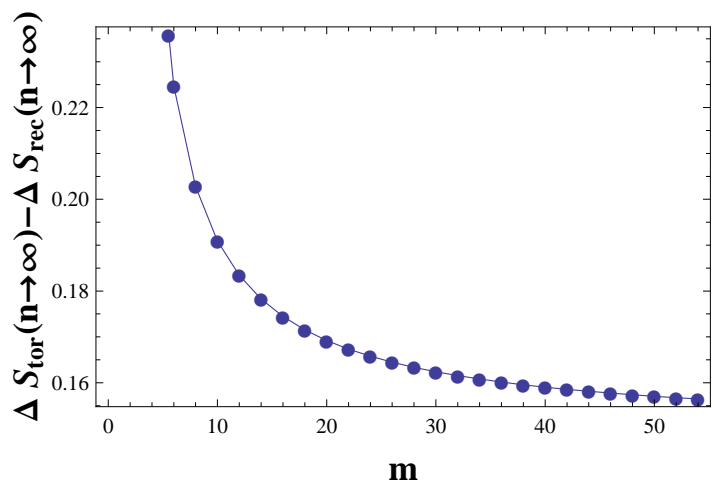

FIG. 9: The value of $\Delta C(m)=\Delta S_{\text {tor }}(n \rightarrow \infty)-$ $\Delta S_{\text {rec }}(n \rightarrow \infty)$ as a function of the number of rows $m$. $\Delta C(m)$ approaches zero as $m$ goes to infinity.

\section{THE ENTROPY PRINCIPAL OF FUSING TWO SMALL DIMER FILMS INTO ONE BIG DIMER FILM}

The second law of thermodynamics requires that fusing two different types of molecule into one solution would increase the entropy of the system. Two molecules would dissolve into each other much easier if the entropy increased much more. The odd even effect can be understand by the second law of thermodynamics. First, we calculate the entropy before the two molecules meet, $S_{a}$. Then, calculate the entropy after they dissolve in each other, $S_{b}$. The entropy difference in this process, $\Delta S=S_{b}-S_{a}$, determines wether they dissolve into each other or not. The practical liquid crystal molecule is too complicate to accomplish an exact calculation of entropy. We still focus on the ideal dimer film to check if there exist odd even effect when two small films fuse into a big film(Fig. 2).

The entropy is a monotonic logarithmic function of the number of configurations. We use the number of configurations to quantify the entropy of dimer film. The

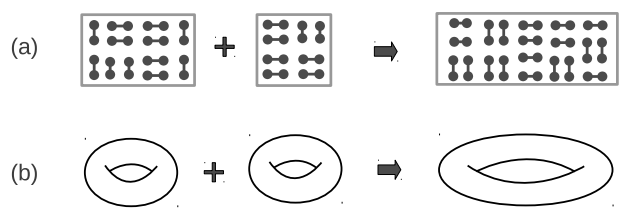

FIG. 10: (a) Fusing two small rectangles with finite size into one big rectangle. (b) Fusing two small toruses into one big torus. We compute how much the entropy increased after the fusion.

rectangle of square lattice has $m$ rows and $n$ columns. The number of rows $m$ is fixed in computation. Only the number of columns $n$ is decomposed as the sum of two smaller numbers: $p$ and $n-p$. The increase rate of possible dimer configurations for fusing two separated film into one is

$$
\Delta Z=\frac{Z_{m, n}-Z_{m, p} Z_{m, n-p}}{Z_{m, n}}
$$

Because $\Delta Z$ is not always positive, if we take the logarithm function of $\Delta Z$ to compute the entropy, the information of negative $\Delta Z$ would be lost. The number of dimer configurations increases(decrease) as entropy increase(decrease). $\Delta Z$ can quantify the variation of entropy.

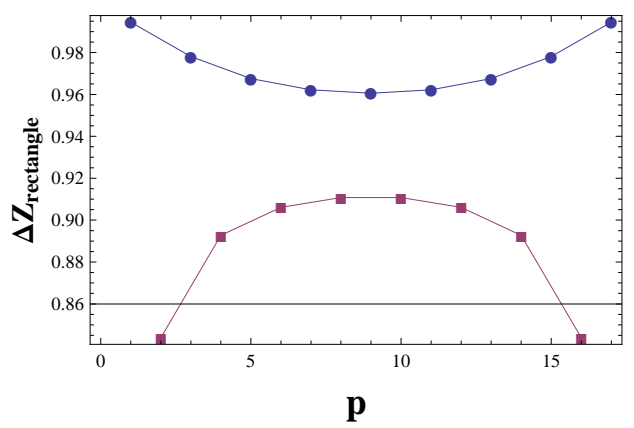

(a)

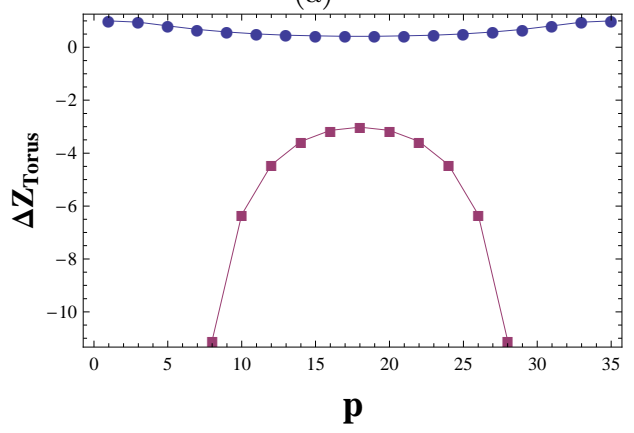

(b)

FIG. 11: (a) The increased number of states after dividing a finite rectangle into two smaller parts. The big rectangle has $m=18$ rows and $n=18$ columns. $m=18$ is invariant. The length of $n$ is divided into two parts, such as $(1,17),(2,16)$, $(3,15), \cdots$, and so forth. (b)The difference of total number states before and after dividing a finite torus with $m=n=36$. 
We took a sequence of pair of two small dimer film with the number of columns, $\{(1, n-1),(2, n-2),(3, n-$ $3), \cdots\}$. The two small films fuse into a big film with $n$ columns. The entropy increase for fusing two dimer films of an odd length is larger than that for fusing two dimer films with an even length(Fig. 11(a)). The entropy gain forms two bands for both rectangle and torus. The upper band is the entropy increase at odd numbers. The lower band is the entropy increase at even number of length. There is a significant difference between rectangle and torus. For rectangle, the entropy increase is positive for both fusing two odd small rectangles and two even rectangles. For torus, the entropy increase for fusing two odd small torus is positive. However fusing two even torus does not increase entropy, instead the entropy is reduced after the fusion (Fig. 11 (b)). According to the second law of thermodynamics, the fusion of two odd rectangles or torus is most favored by entropy. Two even rectangle can fuse into one, but two even torus would repel each other. Topological difference is significant in finite size scale. We doubled the length of the mother torus by keeping $m$ invariant. The gap of the entropy gain between even number and odd number becomes smaller(Fig. 12).

For the two bands of entropy increase, the maximal variation occurs when the two small dimer films is significantly different in length. The minimal entropy increase is situated at the middle point where the two small dimer films have almost identical length. The two spatial dimension parameters play competing roles in controlling the gap between the two bands. If we keep $n$ invariant and doubled $m$, the entropy increase for fusing two odd torus approximate to zero. But entropy reduction for fusing two even torus becomes stronger. The band gap between even and odd grows wider. Therefore increasing $n$ makes smaller band gap, while increasing $m$ enlarge the band gap.

According to the second law of thermodynamic, we may come to the conclusion only two odd toruses of dimer film can fuse into one big torus. If a big torus splits into two small torus, it is most likely two even torus. Fusing two odd rectangles is easier than fusing two even rectangles. This theoretical study maybe is helpful for understanding why two liquid crystal molecules are miscible only when the linker includes odd number of units.

\section{THE CORRELATION OF TWO INTERSECTING LOOPS ON TORUS}

Torus is a manifold generated by sweeping one circle around another circle(Fig. 13 (a)). A torus is equivalent to a rectangle with periodic boundary condition in both of the two spatial dimensions. The two circles, $L_{1}$ and $L_{2}$, are the boundaries of rectangle(Fig. 13 (b)). The entropy of dimer film is reduced when a torus film transforms into a rectangle film. The entropy reduction during this topological transformation quantifies the geometric correlation between $L_{1}$ and $L_{2}$.
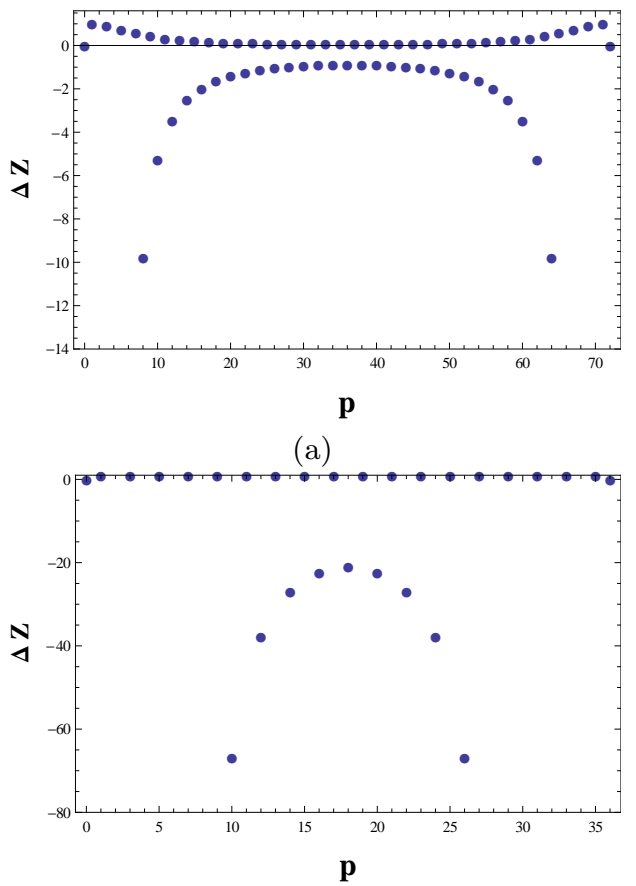

(b)

FIG. 12: (a) The variation of number of states for dividing a finite torus with ( $m=72, n=36)$ and uniting them. Comparing with the finite torus in Fig. [1](b), the number of rows is doubled. (b) The difference of number of states before and after dividing the finite torus with $(m=36, n=72)$. The number of columns is doubled comparing with Fig. 11 (b).

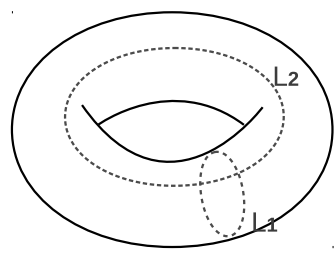

(a)

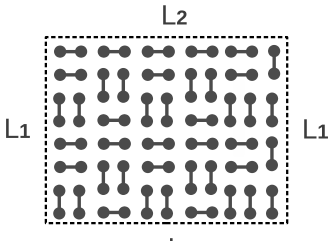

L2 (b)

FIG. 13: (a) A torus with two topologically inequivalent circles, $L_{1}$ and $L_{2}$. (2) A torus covered by dimers can be constructed from a rectangle lattice by taking periodic boundary condition in two spatial dimensions.

In the free fermion theory of dimer model, the correlation of two monomers is defined by the ratio of the total number of configurations with two monomers to the case without two monomers [17] 18] [19]. The two loops are long string of many monomers. Usually the number of dimer configurations of rectangle is smaller than that of torus. For a finite lattice, the difference of the number of configurations between a torus and a rectangle is small, the subtle information of loop correlation is almost invisible by the definition of the references above. Here we act a logarithm operation on the traditional correlation function to extract out the subtle odd-even effect. The 
correlation of the two intersecting loops is defined as

$$
\begin{aligned}
C\left(L_{1}, L_{2}\right) & =\log \left[\left\langle\psi_{1} \psi_{2} \cdots \psi_{n} \psi_{o} \psi_{1} \psi_{2} \cdots \psi_{m}\right\rangle\right], \\
& =\log \left[\left\langle L_{1} L_{2}\right\rangle\right]
\end{aligned}
$$

Loop $L_{1}$ is a string of $n+1$ monomers, $\psi_{1} \psi_{2} \cdots \psi_{n} \psi_{\text {o }}$. Loop $L_{2}$ covers $m$ monomers, $\psi_{1} \psi_{2} \cdots \psi_{m}$, and the monomer at the intersecting point, $\psi_{o}$. This loop correlation function quantifies the correlation among $(n+m+1)$ fermions. Here $C\left(L_{1}, L_{2}\right)$ is actually the entropy difference between rectangle and torus,

$$
C\left(L_{1}, L_{2}\right)=\log \left[\frac{Z_{r e c}}{Z_{t o r}}\right]=S_{r e c}-S_{t o r} .
$$

We keep the total number of fermions expanding the two loops as constant, $L_{1}+L_{2}=n+m+1=$ const. Generally speaking, the geometric correlation between the two loops is negative. If one circle becomes larger, the other circle must be smaller.

The correlation of two loops shows odd-even dependence on the length of the loop(Fig. 14). First, we keep $m+n=48$, and both $(m, n)$ are even. The correlation is weak at small $n$ and large $n$, the maximal correlation is around the middle, $n=24$. Then, we still keep $m+n=48$, but modify $n$ and $m$ in a sequence of odd numbers. The strong correlation now appears at small $n$ and large $n$. Around $n=23$ is the minimal correlation which is still larger than the maximal value for even case. We come to the conclusion the correlation between two loops with odd number of length is stronger than the correlation of loops with even number of length. The correlation has a finite gap between the odd and the even case. When the length of the two loops grows to infinity, this gap becomes zero. The odd even effect of loop correlation holds for other hybrid cases: (1) $\mathrm{m}$ is even and $\mathrm{n}$ is odd. The correlation curve inherits one half of the even case and one half of the odd case. For $m+n=49$, the maximal correlation appear at small $n$, and the minimal correlation appears at large $n$. (2) $m$ is odd and $n$ is even(Fig. 14). For $m+n=49$, the correlation curve is the mirror image of case (1) reflected by the vertical axis $n=23$.

The loop correlation function $C\left(L_{1}, L_{2}\right)$ define the geometric correlation of the two finite loops. The two free fermion loops also obey a topological constraint as long as the fermion loop does not break into an open chain.

\section{THE MELTING TEMPERATURE OF LONG POLYMER'S FILM}

The melting temperature for a film of long polymers can be computed by the same critical temperature Eq. (11) for dimers. First we need to count the number of external bonds. Then we count all possible ways to cover the lattice by trimer(3-connected monomers), quadramer(4-connected monomers), pentagomer(5-connected monomers), and so

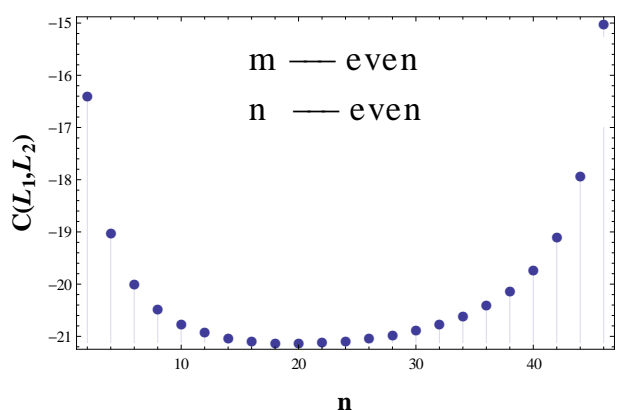

(a)

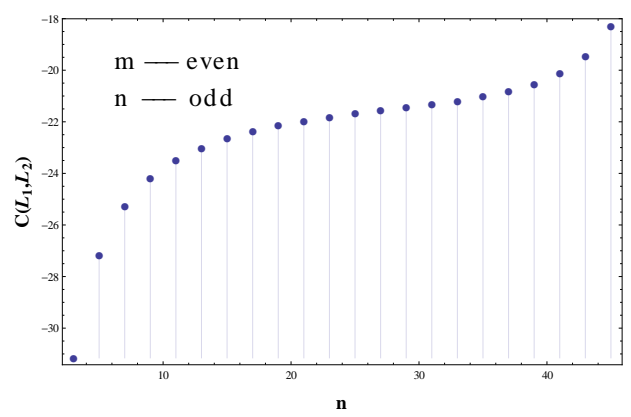

(b)

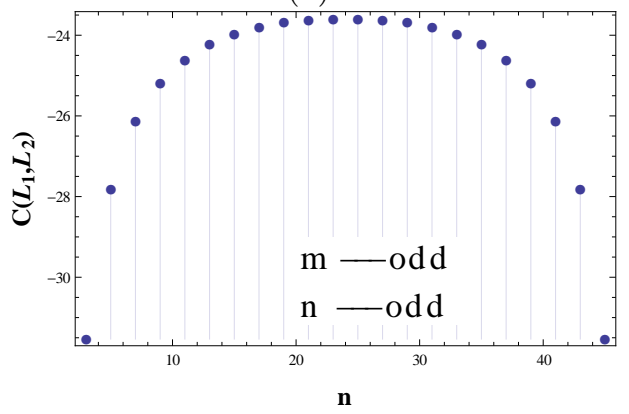

(c)

FIG. 14: (a)The sum of the two loops is constant, $L_{1}+L_{2}=$ 49 , i.e., $\mathrm{m}+\mathrm{n}=48$. Both $m$ and $n$ are even number varying from 2 to 48. (b) The total length of the two loop is 50, $\mathrm{m}+\mathrm{n}=49$. The length of $L_{2}$ varies as following, $(2,4,6, \cdots, 48)$. The corresponding length of $L_{2}$ is $(47,45,43, \cdots, 3,1)$. (c) The sum of the two loop is $49, \mathrm{~m}+\mathrm{n}=48$. Both $m$ and $n$ varies from odd number to odd number.

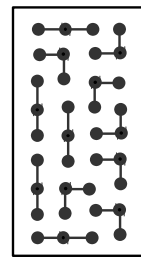

$\mathrm{C}_{3} \mathrm{H}_{6}$

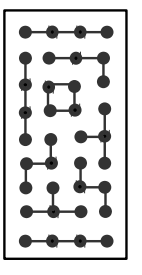

$\mathrm{C}_{4} \mathrm{H}_{10}$

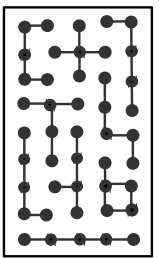

$\mathrm{C}_{5} \mathrm{H}_{12}$

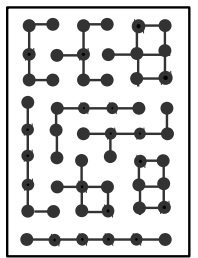

$\mathrm{C}_{6} \mathrm{H}_{14}$
FIG. 15: Covering of a finite square lattice by long polymers. The length of the mathematical polymer showed above is 3 , $4,5,6$. The mathematical polymer represents the n-alkanes $C_{n} H_{2 n+2}$. Soft polymer can bend into different elementary configurations. 
on(Fig. 15). However it is a difficult mathematical problem to exactly count all possible polymer coverings by long polymers. So far we only have the exact solution of dimer covering problem. For the simplest case that the polymer is rigid enough to keep the shape of a straight line, the entropy of longer polymer would be smaller than the entropy of shorter polymers for covering the same area. We can always cut the longer polymers shorter, as a result, the number of possible configurations would increase. According to Eq. (11), smaller entropy induces larger melting temperature. Although the number of external bonds also decreases for longer polymer, it falls far behind the decreasing speed of entropy. Qualitatively we can predict that the melting temperature would increase when the length of the polymer increases. In mind of the experimental observation of odd-even effect, we believe the exact entropy of long polymer covering would demonstrate odd-even effect. In fact, it is possible to measure the melting temperature of a film in lab. So experiment maybe is another way for solving mathematical problems.

In reality, the n-alkanes $C_{n} H_{2 n+2}$ is not very rigid for large $n$. The soft chain would bend into different configurations(Fig. 15). In that case, the entropy of longer polymer maybe is larger than shorter polymers. Then we would meet the anomalous odd-even effect. For a more complicate case, if we mix polymers of different length, it is almost impossible to get exact mathematical counting of all possible coverings. But we can divide the polymers into two classes: even-polymer and odd-polymers. The odd-even effect would still play a role.

\section{SUMMARY}

Odd-even effect is a common phenomena in finite size system. If certain degree of freedom of a system is highly confined in finite scale, the physical observable would strongly depends on the confined dimensions. We study how the melting temperature and entropy depend on the finite width of a long dimer film. As a mathematical modeling of the melting process of a dimer film, we assumed there is a weak external bonding between neighboring dimers and defined the transition between a solid dimer phase and a liquid dimer phase. Kasteleyn's method was used for computing the entropy of the liquid dimer phase. The melting temperature curve connecting the odd width is above that connecting the even width. While the entropy curve threading the even width is above that of the odd width. The odd even effect also exist in the fusion of two smaller dimer films into a bigger dimer film. The entropy increase for fusing two odd rectangular film is bigger than that for fusing two even rectangular film. For torus film, only fusing two torus films with odd number of length increase the entropy. Fusing two even torus films into a big torus film experiences an entropy decrease. This odd-even effect is consistent with the second law of thermodynamics. The entropy of a system tends to increase during a physical process. The correlation function of two loops on torus also depends on the oddeveness of their length. The correlation of two odd loops is stronger than the correlation of two even loops.

The odd even effect is diminished when the finite width grows longer until thermal dynamic limit. The melting temperature approaches to a constant value. We derived the equation of melting temperature in thermodynamic limit by studying a growing dimer film. The melting temperature is proportional to the external bonding energy and the inverse of entropy per site. This equation has no dependence on boundary condition. The difference between a torus and a rectangle decays to zero as the width goes to infinity. The general equation for computing melting temperature of dimer film also hold for longer polymer, such as trimer, tetramer, and so on. However how to exactly count all the possible configurations of trimer covering or tetramer covering on square lattice is an unsolved mathematical problem. We conjecture that the melting temperature computed by an extended dimer film model would still demonstrate odd-even dependence on the length of polymers. There might be a significant difference between two dimensional dimer film model and three dimensional dimer powder model. Further study will be performed in the future.

\section{ACKNOWLEDGMENT}

This work is supported by the Fundamental Research Foundation for the Central Universities and National Natural Science Foundation of China(11304062).
[1] J. M. Kosterlitz and D. J. Thouless, J. Phys. C 6, 1181 (1973). P. M. Chaikin and T. C. Lubensky, Principles of Condensed Matter Physics (Cambridge Univ. Press, Cambridge, 1995).

[2] C. F. Chou, A. J. Jin, S. W. Hui, C. C. Huang, J. T. Ho, Science, 280, 1424, (1998).

[3] F. L. Chen, A. M. Jamieson, Liq. Cryst. 15(2), 171 (1993).

[4] A. Baeyer, Ber. Dtsch. Chem. Ges., 10, 1286 (1877). D. Vorländer, Z. Phys. Chem. 126, 449 (1927).
[5] R. Boese, H. Ch. Weiss, D. Bläser, Angew. Chem., 111, 1042 (1999);

[6] C. Hägele, E. Wuckert, S. Laschat and F. Giesselmann, ChemPhysChem, 10, 1291 (2009).

[7] A. P. Malanoski and P. A. Monson, J. Chem. Phys. 110, 664 (1999).

[8] A. P. Malanoski and P. A. Monson, J. Chem. Phys. 107, 6899 (1997).

[9] K. Yoon, D. G. Chae, T. Ree, F. H. Ree, J. Chem. Phys. 74, 1412, (1981). 
[10] R. Kenyon, An introduction to the dimer model, arxiv: math/0310326v1, (2003)

[11] P. W. Kasteleyn, Physica Amsterdam 27, 1209 (1961); J. Math. Phys. 4, 287 (1963).

[12] C. Yokoi, J. Nagle, and S. Salinas, J. Stat. Phys. 44, 729 (1986). Y. Jiang and T. Emig, Phys. Rev. B 73, 104452, (2006).

[13] Z. C. Ou-Yang, W. Helfrich, Phys. Rev. Lett. 59, 2486, (1987). Phys. Rev. A, 39, 5280, (1989).

[14] Z. C. Ou-Yang, Phys. Rev. A, 41, 4517, (1990).
[15] A. S. Rudolph, B. R. Ratna, B. Kahn, Nature, 352, 52, (1991). Z. Lin, et al, Langmuir, 10, 1008, (1994).

[16] S. Samuel, J. Math. Phys. 21, 2806 (1980).

[17] R. Moessner, and S. L. Sondhi, Phys. Rev. B 62, 14122, (2002).

[18] P. Fendley, R. Moessner, and S. L. Sondhi, Phys. Rev. B 66, 214513 (2002).

[19] W. J. Tzeng and F. Y. Wu, J. Stat. Phys. 10, 671 (2003). 